\title{
Lack of Synapsin I Reduces the Readily Releasable Pool of Synaptic Vesicles at Central Inhibitory Synapses
}

\author{
Pietro Baldelli, ${ }^{1,2}$ Anna Fassio, ${ }^{1,2}$ Flavia Valtorta, ${ }^{3,4}$ and Fabio Benfenati ${ }^{1,2}$ \\ ${ }^{1}$ Department of Neuroscience and Brain Technologies, The Italian Institute of Technology, 16163 Genova, Italy, ${ }^{2}$ Department of Experimental Medicine, \\ Section of Physiology, University of Genova, 16132 Genova, Italy, and ${ }^{3}$ S. Raffaele Scientific Institute, Vita-Salute University and ${ }^{4}$ IIT Unit of Molecular \\ Neuroscience, 20132 Milano, Italy
}

Synapsins (Syns) are synaptic vesicle (SV) phosphoproteins that play a role in neurotransmitter release and synaptic plasticity by acting at multiple steps of exocytosis. Mutation of SYN genes results in an epileptic phenotype in mouse and man suggesting a role of Syns in the control of network excitability. We have studied the effects of the genetic ablation of the SYN1 gene on inhibitory synaptic transmission in primary hippocampal neurons. Inhibitory neurons lacking SynI showed reduced amplitude of IPSCs evoked by isolated action potentials. The impairment in inhibitory transmission was caused by a decrease in the size of the SV readily releasable pool, rather than by changes in release probability or quantal size. The reduction of the readily releasable pool was caused by a decrease in the number of SVs released by single synaptic boutons in response to the action potential, in the absence of variations in the number of synaptic contacts between couples of monosynaptically connected neurons. The deletion of SYN1 did not affect paired-pulse depression or post-tetanic potentation, but was associated with a moderate increase of synaptic depression evoked by trains of action potentials, which became apparent at high stimulation frequencies and was accompanied by a slow down of recovery from depression. The decreased size of the SV readily releasable pool, coupled with a decreased SV recycling rate and refilling by the SV reserve pool, may contribute to the epileptic phenotype of SynI knock-out mice.

Key words: synapsin; GABA release; synaptic transmission; knock-out mice; quantal analysis; synaptic plasticity; epilepsy

\section{Introduction}

Synapsins (Syns) are major synaptic vesicle (SV)-specific phosphoproteins that play multiple roles in synaptic transmission and plasticity (Hilfiker et al., 1999; Baldelli et al., 2006). Three distinct $S Y N$ genes (SYN1, SYN2, and SYN3) give rise in mammals to several differentially spliced isoforms whose expression is developmentally regulated, SynIII being the most abundant isoform at early stages of neuronal development and SynI the most abundant isoform in mature neurons (Ferreira et al., 2000). Synapsins have a major predocking action by controlling SV transitions from the reserve pool (RP) to the readily releasable pool (RRP) through a phosphorylation-dependent regulation of SV-actin interactions (Ceccaldi et al., 1995; Hosaka et al., 1999; Chi et al., 2001, 2003; Bonanomi et al., 2005; Menegon et al., 2006). Moreover, previous data indicate that Syns also play a role in the final postdocking steps of exocytosis including SV priming and fusion with the presynaptic membrane (Hilfiker et al., 1998; Humeau et

Received July 11, 2007; revised 0ct. 16, 2007; accepted 0ct. 17, 2007.

This work was supported by PRIN 2006 grants and research grants from the Ministry of the University (P.B., A.F., F.B.), the Fisher (enter for Alzheimer disease Research (F.B.), Telethon-Italy Grant GCP05134, the Cariplo Foundation, and grants from Fondazione Mariani (F.B., F.V.). We thank Drs. Paul Greengard (The Rockefeller University, New York, NY) for providing us with the mutant mouse strains and for invaluable discussions and help, Hung-Teh Kao (New York University, Orangeburg, NY) and Egidio D'Angelo (University of Pavia, 27100 Pavia, Italy) for helpfu discussions, and Silvia Casagrande (University of Genova, Genova, Italy) for precious help with hippocampal cultures.

Correspondence should be addressed to Dr. Pietro Baldelli, Department of Experimental Medicine, University of Genova, Viale Benedetto XV, 3, 16132 Genova, Italy. E-mail: pietro.baldelli@unige.it.

D0I:10.1523/JNEUROSCI.3151-07.2007

Copyright $\odot 2007$ Society for Neuroscience $\quad$ 0270-6474/07/2713520-12\$15.00/0 al., 2001; Hilfiker et al., 2005; Fassio et al., 2006; Hvalby et al., 2006; Sun et al., 2006).

Genetic deletion of single or multiple SYN genes (with the notable exception of SYN3) (Feng et al., 2002) produces a severe epileptic phenotype characterized by partial, secondarily generalized seizures that appear after 2-3 months of age and become progressively more intense (Rosahl et al., 1995; Gitler et al., 2004). Interestingly, a familial X-linked epilepsy has been recently reported to be associated with a nonsense mutation in the human SYN1 gene (Garcia et al., 2004). The epileptic phenotype suggests that SynI has a role in the control of the excitability of cortical networks.

At mammalian excitatory synapses, SYN deletion reduces the size of the RP of SVs (Li et al., 1995; Takei et al., 1995; Ryan et al., 1996; Siksou et al., 2007) and alters short-term plasticity (Rosahl et al., 1995; Gitler et al., 2004; Hvalby et al., 2006; Kielland et al., 2006; Sun et al., 2006) without affecting release evoked by isolated action potentials. On the contrary, inhibitory synapses lacking SynI (Terada et al., 1999), SynIII (Feng et al., 2002), or SynI/ SynII/SynIII (Gitler et al., 2004) showed a decrease in the amplitude of the IPSCs evoked by single action potentials (eIPSCs), suggesting the involvement of postdocking mechanisms. However, the role of Syns in the control of the quantal release parameters at inhibitory synapses has never been investigated.

Using patch-clamp recordings on cultured hippocampal neurons from SynI knocked out (KO) mice, we observed a reduction in the amplitude of eIPSCs with respect to wild type (WT) neu- 
rons. Such effect was found also at single synaptic boutons and was associated with a parallel decrease in the number of release sites, in the absence of changes in either quantal size or release probability. The lack of SynI was associated with increased synaptic depression and slower recovery from depression, an effect that was apparent only at high stimulation frequency and was occluded by lowering intraterminal $\mathrm{Ca}^{2+}$. The results indicate that the impairment of inhibitory transmission observed in Syn $\mathrm{KO}$ mice is contributed by significant changes in the postdocking events of exocytosis and can play a direct role in the pathogenesis of the epileptic phenotype.

\section{Materials and Methods}

Cell cultures. Syn I KO mice were generated by homologous recombination (Chin et al., 1995). Offspring of littermates of WT and homozygous SynI KO mice were used throughout. All experiments were performed in accordance with the guidelines established by the National Council on Animal Care and approved by the local Animal Care Committee of the University of Genova. Mice were killed by inhalation of $\mathrm{CO}_{2}$, and embryonic day 17 (E17) embryos were removed immediately by cesarean section. Removal and dissection of hippocampus, isolation of neurons and culturing procedures were as those previously described (Baldelli et al., 2000). The isolated hippocampal neurons were plated at low density $\left(100\right.$ cells $\left./ \mathrm{mm}^{2}\right)$ and maintained in a culture medium consisting of Neurobasal, B-27 $(1: 50 \mathrm{v} / \mathrm{v})$, glutamine $(1 \% \mathrm{w} / \mathrm{v})$, penicillin-streptomycin $1 \%$ (all from Invitrogen, Carlsbad, CA). Every $7 \mathrm{~d}$ for 3-4 weeks, a half-volume medium replacement was performed. Hippocampal autaptic cultures were prepared as described previously (Bekkers and Stevens, 1991) with slight modifications. Briefly, dissociated neurons were plated at very low density $\left(20\right.$ cells $\left./ \mathrm{mm}^{2}\right)$ on microdots $(40-300 \mu \mathrm{m}$ in diameter) obtained by spraying a mixture of poly-D-lysine $(0.4 \mathrm{mg} / \mathrm{ml} \mathrm{w} / \mathrm{v})$ and collagene $(0.25 \mathrm{mg} / \mathrm{ml})$ on dishes which had been pretreated with $0.15 \%$ agarose. Both glial cells and single autaptic neurons were present under this culture condition. Electrophysiological experiments were performed on 7-28 d in vitro (DIV) neurons.

Current recordings, data acquisition, and analysis. Patch electrodes, fabricated from thick borosilicate glasses (Hilgenberg, Mansfield, Germany), were pulled and fire-polished to a final resistance of 3-4 M $\Omega$. Monosynaptic GABAergic IPSCs were investigated either in pairs of monosynaptically connected neurons or in autaptic neurons of 7-28 DIV. Whole-cell patch-clamp recordings from presynaptic and postsynaptic neurons were simultaneously performed using an EPC-10 double amplifier (HEKA Elektronic, Lambrecht, Germany). For the evaluation of synaptic depression and recovery from depression, presynaptic stimuli were delivered through a glass pipette $(1 \mu \mathrm{m}$ tip diameter) filled with Tyrode solution and placed in contact with the soma of the GABAergic interneuron in a loose-seal configuration. In this case, current pulses of $0.1 / 0.3 \mathrm{~ms}$ and variable amplitude $(10-45 \mu \mathrm{A})$ delivered by an isolated pulse stimulator (model 2100; A-M Systems, Carlsburg, WA) were required to induce eIPSCs with short latency $(2-4 \mathrm{~ms})$. Miniature IPSCs (mIPSCs) and eIPSCs were acquired at $5-20 \mathrm{kHz}$ sample frequency and filtered at half the acquisition rate with an 8-pole low-pass Bessel filter. Recordings with leak currents $>100 \mathrm{pA}$ or series resistance $>20 \mathrm{M} \Omega$ were discarded. Data acquisition was performed using PatchMaster programs (HEKA Elektronic). The mIPSCs analysis was performed by using the Minianalysis program (Synaptosoft, Leonia, NJ). The amplitude and frequency of mIPSCs were calculated using a peak detector function with a threshold amplitude set at $4 \mathrm{pA}$ and a threshold area at $50 \mathrm{~ms} \cdot \mathrm{pA}$. All the experiments were performed at room temperature $\left(22-24^{\circ} \mathrm{C}\right)$. Data were expressed as means \pm SE for number of cells $(n)$. One-way ANOVA followed by either unpaired Student's $t$ test or Tukey's multiple comparison test were used and $p$ values $<0.05$ were considered significant.

Solutions and drugs. mIPSCs and eIPSCs were recorded by superfusing the whole-cell clamped postsynaptic neuron with a Tyrode solution containing (in mM) $2 \mathrm{CaCl}_{2}, 150 \mathrm{NaCl}, 1 \mathrm{MgCl}_{2}, 10 \mathrm{HEPES}, 4 \mathrm{KCl}, 10$ glucose, pH 7.4. D-(-)-2-amino-5-phosphonopentanoic acid (D-AP5; $50 \mu \mathrm{M}$ ), 6-cyano-7-nitroquinoxaline-2,3-dione (CNQX; $10 \mu \mathrm{M}$ ), and CGP58845 (5 $\mu \mathrm{M})$ (Tocris, Bristol, UK) were added to the Tyrode solu- tion to block NMDA, non-NMDA, and $\mathrm{GABA}_{\mathrm{B}}$ receptors, respectively. In some cases, the nonselective glutamate receptor antagonist kynurenic acid (1 mM; Sigma, St. Louis, MO) was used to block excitatory transmission. Tetrodotoxin (TTX, $0.3 \mu \mathrm{M}$; Tocris) was added to block spontaneous action potentials propagation. Subsaturating concentrations of $\mathrm{Cd}^{2+}$ were used to reduce neurotransmitter release probability. Neurons were constantly superfused through a gravity system constituted by a multibarrelled pipette with a single outlet and five inlets controlled by solenoid electrovalves (Lee, Westbrook, CO) operated by a computer (Baldelli et al., 2002). The perfusion solution could be changed rapidly $(50-60 \mathrm{~ms})$ and applied for controlled periods. The tip of the perfusion pipette $(100-200 \mu \mathrm{m}$ in diameter) was placed at $40-80 \mu \mathrm{m}$ from the soma. The standard internal solution was (in $\mathrm{mm}$ ) $90 \mathrm{CsCl}, 20$ tetraethylammonium (TEA)-Cl, 10 EGTA, 10 glucose, $1 \mathrm{MgCl}_{2}$, 4 ATP, 0.5 GTP, and 15 phosphocreatine, $\mathrm{pH}$ 7.4. $\mathrm{K}^{+}$was substituted for $\mathrm{Cs}^{+}$and TEA ${ }^{+}$ in the pipette solution to block outward $\mathrm{K}^{+}$currents. QX-314 $(N-(2,6-$ dimethylphenyl carbamoylmethyl)triethylammonium bromide; $10 \mathrm{~mm}$; Tocris) was added to block $\mathrm{Na}^{+}$currents when extracellular stimulations were used.

Calculation of IPSC amplitude during high-frequency repetitive stimulation and paired-pulse stimulation protocols. eIPSCs were inspected visually and only those not contaminated by spontaneous activity were considered. When the stimulation time interval was shorter than the time needed for an eIPSC to return to baseline, eIPSCs overlapped partially or massively depending on the stimulation frequency. During a train stimulation protocol, the baseline of each event was defined as the final value of the decay phase of the preceding eIPSC and the amplitude of eIPSC number $n$ was calculated by subtracting the residual amplitudes of the previous eIPSCs from its peak value. To analyze the paired-pulse ratio (PPR), two brief supraliminar depolarizing pulses were applied to the presynaptic neuron at $20-2500 \mathrm{~ms}$ intervals. For each couple of eIPSCs, PPR was calculated from the equation PPR $=100 \times\left(I_{1}-I_{2}\right) / I_{1}$, where $I_{1}$ and $I_{2}$ are the amplitudes of the eIPSCs evoked by the conditioning and test stimuli, respectively (Mallart and Martin, 1967). The amplitude of $I_{2}$ was determined as the difference between the $I_{2}$ peak and the corresponding value of $I_{1}$ calculated by monoexponential fitting of the eIPSC decay (Jensen et al., 1999). Because of the high intrinsic variability of PPR, the mean PPR was calculated from the responses to at least eight paired-pulse stimulation protocols for each interpulse interval.

Multiple probability fluctuation analysis. The analysis of the three main determinants of synaptic function, namely the average amplitude of the postsynaptic response to a neurotransmitter quantum $\left(Q_{\mathrm{av}}\right)$, the average probability of a SV release from a release site $\left(\operatorname{Pr}_{\mathrm{av}}\right)$, and the number of independent release sites $(N)$, was performed by building variancemean (V-M) plots (Clements and Silver, 2000). $Q_{\mathrm{av}}, \operatorname{Pr}_{\mathrm{av}}$, and $N$ were derived from the parabolic relationship between eIPSC variance $\left(\sigma^{2}\right)$ and mean postsynaptic current amplitude $\left(I_{\mathrm{av}}\right): \sigma^{2}=A I_{\mathrm{av}}-B I_{\mathrm{av}}{ }^{2}$, recorded under different release probability conditions by assuming that $I_{\mathrm{av}}=N Q_{\mathrm{av}} \operatorname{Pr}_{\mathrm{av}}$ (Silver et al., 1998; Reid and Clements, 1999). The free parameters A (initial slope) and B (curvature of the parabola) were iteratively adjusted to optimally fit the $\mathrm{V}-\mathrm{M}$ plots and used to calculate a weighted mean of $P r_{\mathrm{av}}$ and $Q_{\mathrm{av}}$ and a lower limit for the number of independent release sites, $N_{\text {min }}: N_{\text {min }}=1 / B ; Q_{\mathrm{av}}=A /\left(1+C V_{i}^{2}\right) ; P r_{\mathrm{av}}=$ $I_{\mathrm{av}}(B / A)\left(1+C V_{i}^{2}\right)$, where $C V_{i}$ is the coefficient of variation of mIPSC amplitudes at an individual release site. In our case, $C V_{i}$ is $0.36 \pm 0.01$ $(n=6)$ in WT neurons and $0.35 \pm 0.02(n=6)$ in KO cells. Pr was varied adding increasing concentrations of $\mathrm{Cd}^{2+}$ to the external solution $(0,2$, $4,6 \mu \mathrm{M}) . I_{\mathrm{av}}$ and $\sigma^{2}$ were calculated over a stable epoch of 30-150 events after the wash-in of each extracellular solution. Presynaptic stimulation continued during the wash-in at $0.1 \mathrm{~Hz}$. After the solution exchange, the eIPSC amplitude remained stable throughout the subsequent analysis epoch. The variance attributable to the recording noise was estimated in the region before the test pulse, and was subtracted from the eIPSC variance. A zero point was included in each $\mathrm{V}-\mathrm{M}$ plots to indicate that the noise variance was subtracted.

Cumulative eIPSC amplitude analysis. The size of the RRP of synchronous release $\left(\mathrm{RRP}_{\text {syn }}\right)$ and the probability that any given $\mathrm{SV}$ in the RRP will be released ( $\mathrm{P}_{\mathrm{ves}}$, to distinguish it from $P r_{\mathrm{av}}$, see above) were calculated using the cumulative amplitude analysis (Schneggenburger et al., 
2002). $R R P_{\text {syn }}$ was determined by summing up peak IPSC amplitudes during 40 repetitive stimuli applied at a frequency of $40 \mathrm{~Hz}$. This analysis assumes that depression during the steady-state phase is limited by a constant recycling of SVs and equilibrium occurs between released and recycled SVs (Schneggenburger et al., 1999). The number of data points to include in the linear fitting of the steady-state phase was evaluated by calculating the best linear fit including the maximal number of data points starting from the last data point (i.e., from the 40th IPSC). The cumulative amplitude profile showed the best linear course after the first 15 stimuli, in the range of $400-1000 \mathrm{~ms}$ in both WT $(n=8)$ and $\mathrm{KO}(n=8)$ neurons. Thus, the last 25 data points were fitted by linear regression and back-extrapolated to time 0 (see Fig. $5 B)$. The intercept with the $y$-axis gave the $\mathrm{RRP}_{\text {syn }}$ and the ratio between the first eIPSC amplitude $\left(I_{1}\right)$ and $\mathrm{RRP}_{\text {syn }}$ yielded the $\mathrm{P}_{\mathrm{ves}}$. The total number of SVs ready for release (Nsyn) was determined as the ratio between $\mathrm{RRP}_{\text {syn }}$ by the mean amplitude of mIPSCs.

Estimation of $R R P_{\text {total }}$ by hypertonic solution method. The RRP size of the total release (RRPtotal) was estimated by a third independent and well established method, namely the hypertonic stimulation (Rosenmund and Stevens, 1996). Eighteen to 24 DIV autaptic neurons were voltage clamped at $-70 \mathrm{mV}$ and stimulated by a 1 ms voltage step to $20 / 100 \mathrm{mV}$ applied at a frequency of $0.05 \mathrm{~Hz}$, evoking an isolated IPSC. The same neuron was subsequently stimulated with a hypertonic solution (normal extracellular solution with $500 \mathrm{~mm}$ sucrose added). The charge transfer in the transient part of the synaptic current induced by sucrose application was measured as RRP $\mathrm{Rotal}_{\text {. }}$ The $P r_{\text {total }}$ of the isolated IPSC was estimated by dividing the charge transfer of the isolated IPSC by the RRP $\mathrm{R}_{\text {total }}$. All of the experiments were performed constantly superfusing the autaptic neuron with Tyrode external solution. The perfusion solution could be rapidly changed $(50 / 60 \mathrm{~ms})$ and puffs of hypertonic solution were applied over controlled periods of time (5/10 s). The tip of the perfusion pipette $(50 / 100 \mu \mathrm{m})$ was placed close to the soma $(80 / 150 \mu \mathrm{m})$ (see Fig. $7 A)$.

Identification and stimulation of single synaptic boutons. To identify a single bouton, FM1-43 (Invitrogen; $10 \mu \mathrm{M}$ ) was applied in $50 \mathrm{~mm} \mathrm{KCl}$ (supplemented with $10 \mu \mathrm{M}$ CNQX and $50 \mu \mathrm{M}$ D-AP5) to prevent activation of glutamate receptors) for $2 \mathrm{~min}$ and then washed off for $>5 \mathrm{~min}$. Fluorescent images were taken with an inverted Olympus (Tokyo, Japan) IX71 microscope and analyzed with ImageProPlus 5.1 (Media Cybernetics, Bethesda, MD). We chose dye-stained puncta whose intensity was below average, generally avoiding the faintest (which usually failed to support evoked responses) and the brightest (likely to correspond to aggregates of multiple boutons) puncta. Only isolated boutons (distance from nearest neighbor boutons $>5 \mu \mathrm{m}$ ) located at the soma or on first order dendrites were selected for recordings. To obtain evoked release, the stimulating electrode $(1 / 1.5 \mu \mathrm{m}$ tip diameter) containing the bathing solution was placed under visual guidance very close $(\sim 1 \mu \mathrm{m})$ to the selected bouton. After a series of preliminary tests performed to define stimulus duration and intensity able to evoke higher eIPSC amplitudes and lower failure rates (Fedulova et al., 1999), a brief current pulse of 1 $\mathrm{ms}$ at $5 \mu \mathrm{A}$, applied with an isolated pulse stimulator (A-M Systems; model 2100) was selected as the standard single terminal stimulus for all of the experiments (Kirischuk et al., 1999). To disregard the possibility that other boutons in the neighborhood were coactivated by the current pulse, two different tests were performed. First, a small (1 to $2 \mu \mathrm{m})$
B

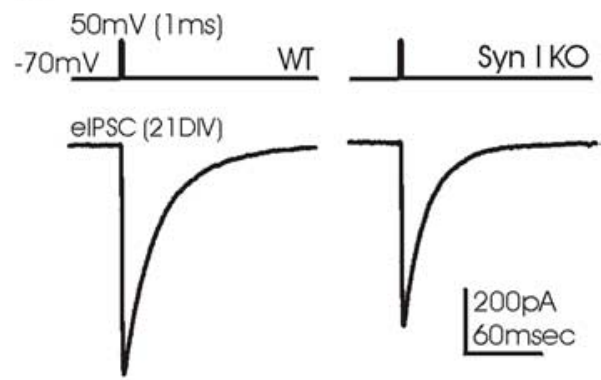

$D$
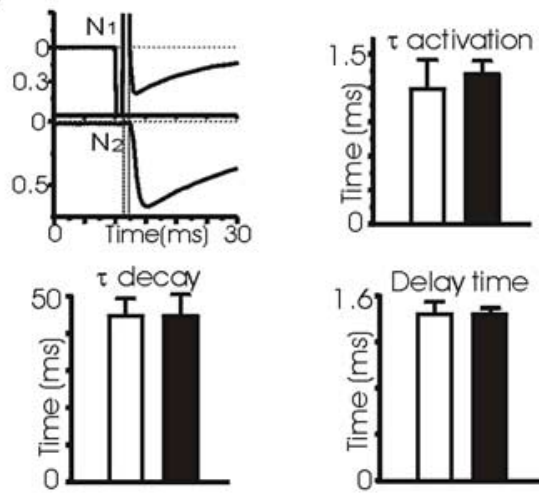

Figure 1. Synl deletion reduces the amplitude but not the kinetics of elPSCs recorded at the soma of whole cell-clamped hippocampal neurons. A, Paired whole-cell recordings were used to record monosynaptic GABAergic elPSCs from primary hip列

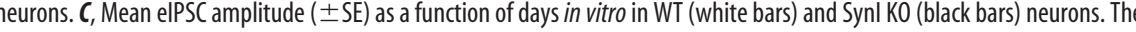
Tukey's multiple comparison test). D, Kinetic analysis of elPSCs. Step depolarization ( $+50 \mathrm{mV} ; 1 \mathrm{~ms}$ ) applied to a WT neuron (N1) (N2) (top left). Rise and decay phases of elPSC were fitted to single exponentials. The mean values ( \pm SE) of activation (top right) and decay (bottom left) time constants $(\tau)$ and delay time (bottom right) revealed that the lack of Synl did not affect IPSC kinetics ( $n=10$ for both WT and Synl K0).

displacement of the stimulation pipette resulted in the reversible disappearance of eIPSCs even when the strongest pulses $(10 \mu \mathrm{A})$ were applied. Second, when the FM1-43 fluorescence intensity was monitored before and after the application of a sustained tetanic stimulation via the stimulus pipette $(30 \mathrm{~s}$ at $20 \mathrm{~Hz} ; 5 \mu \mathrm{A})$, the fluorescence of the spot to which the pipette was directly applied decreased, whereas the fluorescence of neighboring spots was unaffected. TTX (300 nM), D,L-AP5 (50 $\mu \mathrm{M})$, and CNQX $(10 \mu \mathrm{M})$ were included in the bath solution throughout to prevent transmitter release from nonstimulated boutons and to isolate $\mathrm{GABA}_{\mathrm{A}}$ receptor responses. In the presence of TTX and ionotropic glutamate receptor blockade, the focal stimulation of an individual bouton resulted in a postsynaptic chloride current. The GABAergic nature of the synaptic response was identified by its sensitivity to bicuculline $(10 \mu \mathrm{M})$, slow time course and reversal at the chloride equilibrium potential (data not shown). Each synapse was subjected to 100-300 stimulations, repeated at $0.1 \mathrm{~Hz}$. To minimize stimulus artifacts, each trace was subjected to subtraction of the average of 3-6 failure traces in its temporal proximity. Successful events were detected as signals phase-locked with the stimulation (within $3 \mathrm{~ms}$ range). Event detection was performed by setting the peak threshold at $4 \mathrm{pA}$ and the area threshold at $50 \mathrm{~ms} \mathrm{pA}$ (as described for mIPSC detection) using Minianalysis program (Synaptosoft). Events falling below these thresholds were considered as failures. The amplitude distribution of single synapse eIPSCs was analyzed for each individual neuron and density probability histograms were obtained by normalizing the amplitude distributions by the total number of events (Barbara and Takeda, 1996).

Immunocytochemistry. Hippocampal neurons were plated onto glass 
coverslips at the same density used for electrophysiological experiments. Cells were fixed in $4 \%$ paraformaldheyde at $4,7,14$, and 21 DIV and stained using polyclonal antibodies against the vesicular GABA transporter (V-GAT; Synaptic Systems, Göttingen, Germany) followed by Alexa 488 or Alexa 546-conjugated secondary antibodies (Invitrogen). Nuclei were stained with 4',6-diamidino-2-phenylindole (DAPI; Sigma). Neurons in older cultures were counterstained with monoclonal antibodies anti-neuron-specific nuclear protein (NeuN; Millipore, Temecula, CA). The specificity of V-GAT staining for nerve terminals was assessed by double labeling with anti-synaptophysin monoclonal antibodies (Synaptic Systems).

Image analysis. Images were acquired using an IX71 inverted microscope (Olympus) and an ORCA-ER digital CCD camera (Hamamatsu Photonics, Herrsching, Germany). Exposure times were kept constant for all of the acquisitions. Images were taken by randomly focusing on different areas of the dish using the appropriate filter sets for Alexa 488, Alexa 546 and DAPI. Digital images $(672 \times 512$ pixels $)$ from 10 to 19 fields containing one to 10 neurons each were acquired at various ages in vitro from three independent preparations of WT and SynI KO neurons run in parallel. For the quantification of inhibitory synapses on the neuropil, V-GAT-positive puncta were automatically counted within a diameter range of $0.5-2 \mu \mathrm{m}$ using the Image Pro Plus software (Media Cybernetics) and expressed as synaptic puncta/neuron. For the quantification of inhibitory synapses on the soma, V-GAT-positive puncta were manually counted by a blind operator from $150 \mathrm{WT}$ and $222 \mathrm{KO}$ neurons from three distinct preparations run in parallel, normalized by the area of the cell soma and expressed in puncta per squared micrometer.

Immunoblotting. Hippocampal neurons plated at the same density and under the same conditions used for electrophysiological experiments were lysed at 7, 14, and 21 DIV in lysis buffer ( $150 \mathrm{~mm} \mathrm{NaCl}, 1 \% \mathrm{NP}-40$, $0.1 \%$ SDS, 50 mu TrisCl, pH 8.0) in the presence of protease inhibitors. Ten micrograms of protein lysate/sample were loaded onto $10 \%$ SDSpolyacrylamide gels, electrophoretically transferred to nitrocellulose membranes and subjected to immunoblotting with monoclonal or polyclonal antibodies against SynI (mAb 10.22), SynII (mAb 19.21) (Vaccaro et al., 1997), synaptophysin (Synaptic Systems), actin (Sigma) and glutamic acid decarboxylase 67 (GAD67; Millipore) followed by the appropriate peroxidase-conjugated secondary antibodies (Bio-Rad Laboratories, Milan, Italy) and the chemiluminescence detection system (Supersignal West Pico; Pierce Biotechnology, Rockford, IL). Data were quantitatively analyzed by densitomeric scanning of the fluorograms, as described previously (Ferreira et al., 2000).

\section{Results}

Synapsin I deletion reduces the amplitude of eIPSCs, but not their kinetics or short-term plasticity

Double-patch recordings of eIPSCs were used to record stable monosynaptic eIPSCs in paired hippocampal WT and KO neurons at various ages in culture (Fig. $1 A$ ). A significant decrease in the amplitude of IPSCs evoked by isolated action potentials was observed in fully differentiated mutant neurons after 2 weeks in vitro (Fig. $1 B, C)$. On the contrary, at earlier times in vitro $(<13$ DIV), eIPSCs of mutant neurons were undistinguishable from those of WT neurons; thus, all successive recordings were performed at 15-28 DIV. The decrease of eIPSC amplitude was not accompanied by any change in either rise time, decay time or delay time of eIPSC (Fig. 1D).

Synapsins have been implicated in various forms of shortterm synaptic plasticity and their deletion was reported to affect PPR and/or post-tetanic potentation (PTP) of excitatory synapses (Rosahl et al., 1993, 1995), paradigms of temporary change in synaptic strength caused by persistent actions of $\mathrm{Ca}^{2+}$ within the presynaptic terminal (Zucker and Regehr, 2002). However, no data are available thus far on the effects of SynI deletion on PPR and/or PTP of the inhibitory transmission. When hippocampal inhibitory synapses were stimulated by two consecutive stimuli administered at interpulse intervals ranging between
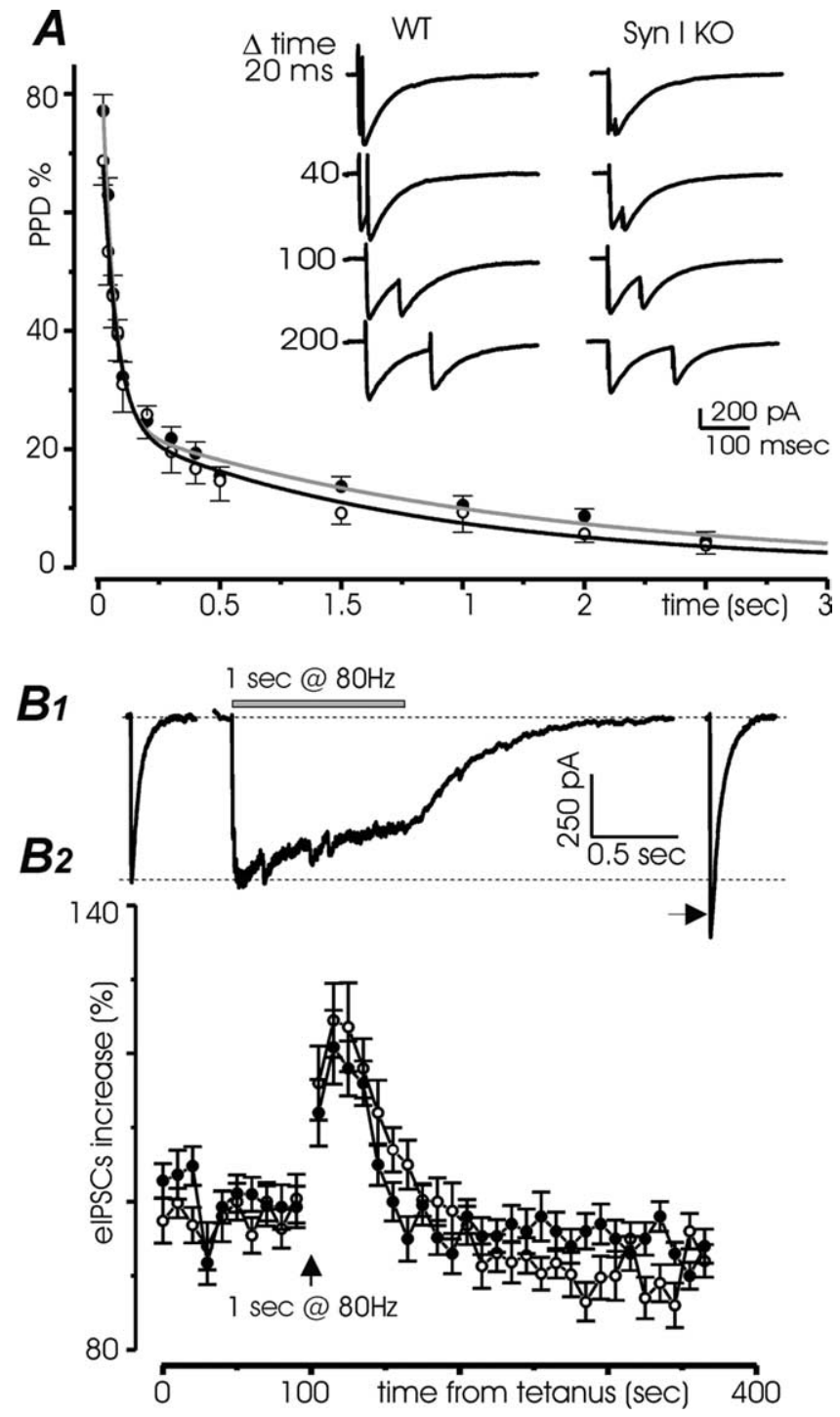

Figure 2. Lack of Synl does not affect paired-pulse depression (PPD) and PTP of elPSCs. $\boldsymbol{A}$, PPD of monosynaptic GABAergic transmission in cultured hippocampal neurons (14-24 DIV) from WT and KO mice. Eight consecutive postsynaptic responses to dual presynaptic stimulation were averaged (stimulation frequency $0.1 \mathrm{~Hz}$ ). The amplitude of the second eIPSC was depressed with respect to the first one and showed greater variability. The percentage of PPD (PPD $\%$ ) was calculated as $\left.100 \times\left[\left(I_{1}-I_{2}\right) / I_{1}\right)\right]$, where $I_{1}$ and $I_{2}$ represent the mean amplitude of the first and second eIPSC averaged over eight consecutive responses, respectively. The mean PPD ( \pm SE) observed in WT (white symbols; $n=20$ ) and Synl KO (black symbols; $n=30$ ) neurons is plotted as a function of the interpulse interval and fitted by a biexponential function (WT, black trace; K0, gray trace). The inset shows dual eIPSCs recorded from whole-cell clamped hippocampal neurons in response to paired stimuli separated by the indicated interpulse intervals ( $\Delta$ time). $\boldsymbol{B}_{1}$, Left, Representative elPSC elicited by single stimuli at $0.1 \mathrm{~Hz}$. Middle, Postsynaptic current during a brief tetanization of the presynaptic neuron ( $1 \mathrm{~s}$ at $80 \mathrm{~Hz}$ ). Right, Second eIPSC after the train (27\% potentiation). $\boldsymbol{B}_{2}$, Mean PTP observed in pairs of neurons (21-28 DIV) from WT (white circles; $n=12$ ) and Synl KO (black circles; $n=12$ ) mice subjected to the train stimulation (arrowhead) as in $\boldsymbol{B}_{\boldsymbol{1}}$. Post-tetanic single elPSCs elicited at $0.1 \mathrm{~Hz}$ were normalized to the pretetanic elPSC level. Peak PTP $(24.4 \pm 0.16$ and $21.08 \pm 0.17 \%$ for WT and K0 neurons, respectively) was reached within $20 \mathrm{~s}$ and decayed to baseline after $\sim 60 \mathrm{~s}$ in both genotypes.

$20 \mathrm{~ms}$ and $2.5 \mathrm{~s}$, no difference in PPR of monosynaptic eIPSCs was found between WT and SynI KO mice (Fig. 2A). A brief tetanization ( $1 \mathrm{~s}$ at $80 \mathrm{~Hz}$ ) of the presynaptic GABAergic neuron was used to induce potentation of subsequent single eIPSCs elicited at $0.1 \mathrm{~Hz}$, as described previously (Jensen et al., 1999). In both WT and SynI KO neurons, PTP reached a maximum of 
$\sim 27 \%$ at the second eIPSC after the stimulus train, with a similar average duration of $\sim 1$ min (Fig. $2 B$ ).

\section{Synapsin I deletion enhances synaptic} depression at GABAergic synapses To evaluate synaptic depression, presynaptic GABAergic neurons were stimulated with trains lasting $110 \mathrm{~s}$ at frequency ranging between 4 and $16 \mathrm{~Hz}$. The progressive decay of eIPSC amplitude during the stimulation train and the subsequent recovery from depression obtained by lowering the stimulation frequency to $0.1 \mathrm{~Hz}$ were both fitted by double-exponential curves. With $4 \mathrm{~Hz}$ stimulation trains, the time course of depression, the steady-state IPSC $\left(I_{\text {ss }}\right)$ and the time course of recovery from depression in WT and KO neurons were closely similar [depression, $\tau_{\text {fast }}, 0.38 \pm 0.03$ and $0.32 \pm 0.03 \mathrm{~s} ; \tau_{\text {slow }}, 23.3 \pm 0.9$ and $17.1 \pm$ $0.8 \mathrm{~s}, I_{\mathrm{ss}}, 0.23 \pm 0.02$ and $0.24 \pm 0.02$; recovery, $\tau_{\text {fast }}, 18.0 \pm 0.6$ and $17.9 \pm 0.7 \mathrm{~s}$, $\tau_{\text {slow }}, 64.1 \pm 4.6$ and $64.7 \pm 6.2 \mathrm{~s}$ for WT $(n=14)$ and $\mathrm{KO}(n=8)$ mice, respectively] (Fig. 3A). The increase in the stimulation frequency enhanced the extent of depression, an effect that was significantly more pronounced in $\mathrm{KO}$ than in WT neurons $\left[8 \mathrm{~Hz}, \tau_{\text {fast }}, 0.21 \pm 0.02\right.$ and $0.24 \pm$ $0.02 \mathrm{~ms}, \tau_{\text {slow }}, 13.2 \pm 0.1$ and $14.4 \pm 0.1 \mathrm{~s}$, $I_{\mathrm{ss}}, 0.21 \pm 0.01$ and $0.16 \pm 0.01$, for WT $(n=13)$ and $\mathrm{KO}(n=17)$ mice, respectively; $16 \mathrm{~Hz}, \tau_{\text {fast }}, 0.13 \pm 0.01$ and $0.10 \pm$ $0.01 \mathrm{~ms}, \tau_{\text {slow }}, 12.5 \pm 0.5$ and $15.9 \pm 0.6 \mathrm{~s}$, $I_{\mathrm{ss}}, 0.15 \pm 0.01$ and $0.06 \pm 0.01$ for WT $(n=20)$ and $\mathrm{KO}(n=20)$ mice, respectively] (Fig. $3 B, C$ ). When the stimulation was returned to $0.1 \mathrm{~Hz}$ after the $16 \mathrm{~Hz}$ tetanus, the rate of recovery in $\mathrm{KO}$ neurons was markedly slowed down with respect to WT neurons $\left[\tau_{\text {fast }}, 8.5 \pm 0.7\right.$ and $24.6 \pm$ $0.9 \mathrm{~ms}, \tau_{\text {slow }}, 208 \pm 17$ and $197 \pm 14 \mathrm{~s}$ for WT $(n=20)$ and KO $(n=20)$ mice, respectively] (Fig. 3C). Thus, although the presence of SynI seems not to be critical in the response to low-to-moderate stimulation frequencies, it plays an important role in keeping the pace with sustained highfrequency stimulations (Fig. $3 E-G$ ).

When the same stimulation protocol shown in Figure $3 C$ was applied on WT and $\mathrm{KO}$ neurons pretreated for $45 \mathrm{~min}$ with $50 \mu \mathrm{M}$ EGTA-AM (Fig. 3D), the effect of Syn I deletion was almost completely occluded. This treatment increased depression and slowed down recovery in WT neurons, although it left substantially unaffected the depression and recovery profiles observed in $\mathrm{KO}$ neurons [depression, $\tau_{\text {fast }}, 0.15 \pm 0.01$ and $0.10 \pm$ $0.01 \mathrm{~s}, \tau_{\text {slow }}, 17.7 \pm 0.5$ and $24.0 \pm 0.8 \mathrm{~s}, I_{\mathrm{ss}}, 0.07 \pm 0.01$ and $0.06 \pm 0.01$; recovery, $\tau_{\text {fast }}, 23.3 \pm 0.8$ and $20.3 \pm 0.9 \mathrm{~s}, \tau_{\text {slow, }}$, $191 \pm 21$ and $222 \pm 23 \mathrm{~s}$ for WT $(n=13)$ and $\mathrm{KO}(n=13)$ mice, respectively]. These data suggest that SynI exerts a positive action on SV exocytosis and recycling that is required only under intense
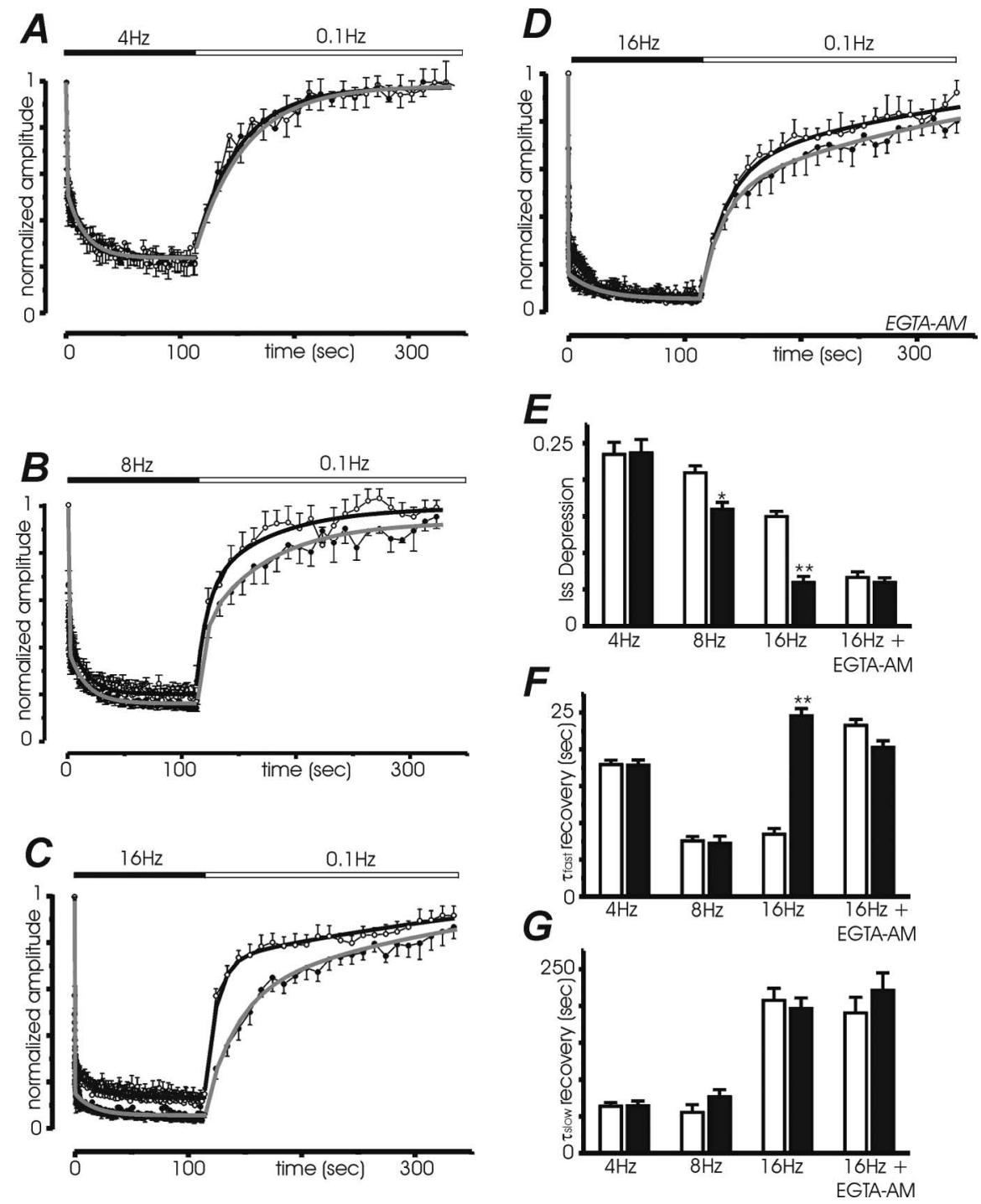

Figure 3. Depression and recovery from depression are altered in Synl KO neurons under high-frequency of stimulation. Synaptic depression and recovery from depression at hippocampal inhibitory synapses activated by trains lasting $110 \mathrm{~s}$ applied at 4,8 or $16 \mathrm{~Hz}$. The recovery from depression was obtained, in all cases, by lowering the stimulation frequency to $0.1 \mathrm{~Hz}$. $\boldsymbol{A}-\boldsymbol{C}, \mathrm{The}$ data were normalized relative to the amplitude of the first eIPSC in the train. The plots of normalized elPSC versus time during repetitive stimulation for $110 \mathrm{~s}$ at $4 \mathrm{~Hz}(\boldsymbol{A} ; n=14$ and 8 for WT and $\mathrm{KO}$ neurons, respectively), $8 \mathrm{~Hz}(\boldsymbol{B} ; n=13$ and 17 for WT and $\mathrm{KO}$ neurons, respectively), or $16 \mathrm{~Hz}$ ( $\boldsymbol{C} ; n=20$ for both WT and $\mathrm{KO}$ neurons) and during the following recovery period are shown (WT, white symbols; KO, black symbols). Only at higher stimulation frequencies, Synl mutant neurons show a stronger synaptic depression and a slowdown of the recovery from depression. $\boldsymbol{D}$, The latter stimulation protocol $(110 \mathrm{~s}$ at $16 \mathrm{~Hz})$ was repeated after treatment with the cell-permeable $\mathrm{Ca}^{2+}$ chelator EGTA-AM ( $50 \mu \mathrm{m}, 45 \mathrm{~min}$ ) which lowers $\mathrm{Ca}^{2+}$ far away from the membrane ( $n=13$ for both WT and KO neurons). Only one experimental point every 10 is plotted in the plateau phase of depression for clarity. Depression and recovery curves were fitted using a biexponential model (WT, black trace; K0, red trace). $\boldsymbol{E}-\boldsymbol{G}$, Mean values ( $\pm \mathrm{SE}$ ) of the resulting normalized steady-state current during depression $\left(\mathrm{I}_{\mathrm{ss}} ; \boldsymbol{E}\right)$, as well as the fast $\left(\tau_{\text {fast }} ; \boldsymbol{F}\right)$ and slow $\left(\tau_{\text {slow }} ; \boldsymbol{G}\right)$ time constants of recovery under the various experimental conditions and for WT (white bars) and KO (black bars) neurons, respectively. ${ }^{*} p<0.05 ;{ }^{* *} p<0.01$ vs WT neurons; Tukey's multiple comparison test.

frequency of stimulation and depends on elevations in intraterminal $\mathrm{Ca}^{2+}$ concentrations.

\section{Synapsin I deletion does not affect spontaneous} neurotransmitter release at inhibitory synapses

To evaluate whether Syn I deletion could affect the GABAergic quantal size, mIPSCs were continuously recorded at the soma of a voltage-clamped neuron held at $-70 \mathrm{mV}\left(V_{\mathrm{h}}\right)$ and locally perfused with a Tyrode solution containing $300 \mathrm{~nm}$ TTX and $1 \mathrm{~mm}$ kynurenic acid to block both spontaneous action potentials and glutamatergic postsynaptic activity (see Materials and Methods). 

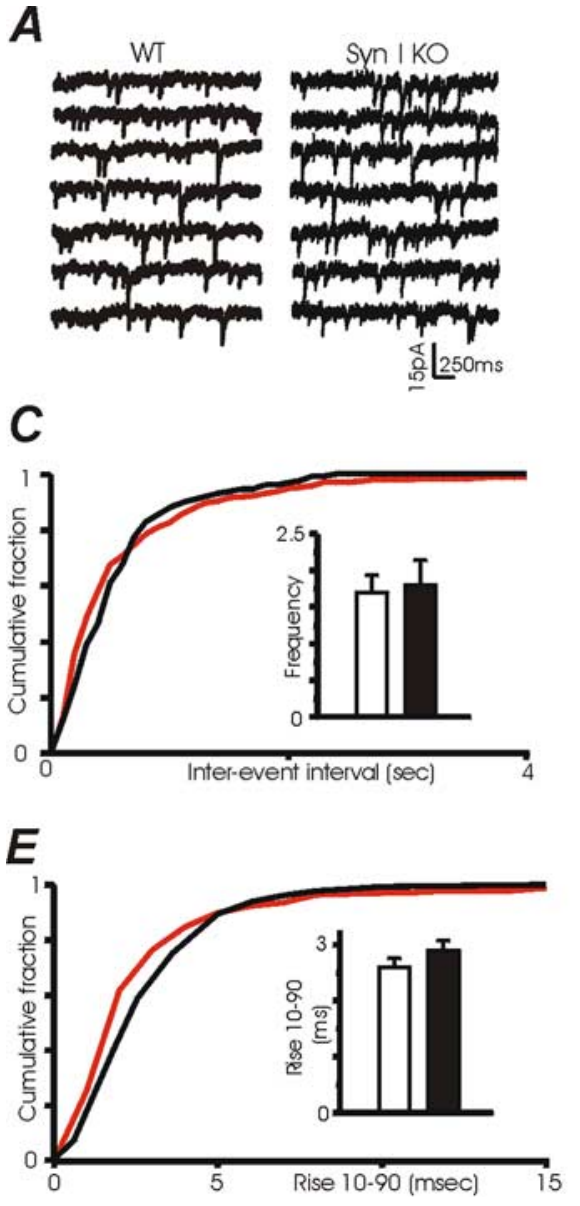

Figure 4. Analysis of mIPSCs. $\boldsymbol{A}$, Consecutive recordings of mIPSCs in WT and Synl KO neurons at 22 DIV. $\boldsymbol{B}$, Cumulative amplitude distribution curves calculated for WT (black trace) and SynI KO (red trace) neurons. The inset shows the mean amplitude ( \pm SE) of mIPSCs for WT (white bar; $n=6$ ) and Synl KO (black bar; $n=6$ ). All cumulative curves and mean values were obtained from 200 to 800 events recorded from each cell. C, Cumulative interevent interval distribution (inset, mean frequency value). D, Cumulative distribution and mean value (inset) of the $50 \%$ decay time. $\boldsymbol{E}$, Cumulative distribution and mean value (inset) of the rise (10-90\%) time. $\boldsymbol{F}$, Cumulative distribution and mean value (inset) of the slope (10-90\%) of the rising phase.

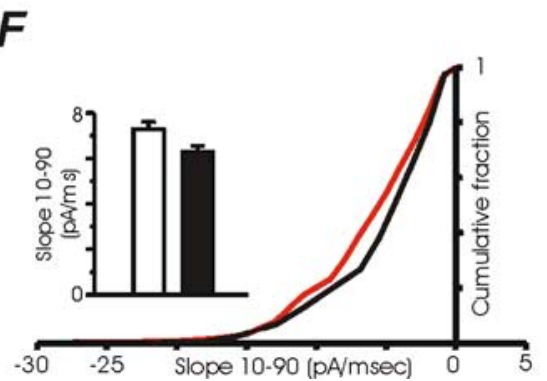

mIPSC activity was closely similar in both WT and $\mathrm{KO}$ neurons (Fig. 4A). The mean mIPSC amplitude (12.46 \pm 0.27 and $12.26 \pm 0.39 \mathrm{pA}, n=6$ for WT and $\mathrm{KO}$ mice, respectively; $p=$ $0.64)$ (Fig. $4 B$ ) and the mean mIPSC frequency $[1.73 \pm 0.22$ and $1.79 \pm 0.33 \mathrm{~Hz}$, for WT $(n=6)$ and $\mathrm{KO}(n=6)$ mice, respectively; $p=0.88$ ] (Fig. $4 C$ ) were not significantly different between the two neuronal genotypes, indicating no change in the rate and quantum content of spontaneous transmitter release. Moreover, there was also no change in the MIPSC waveform, as indicated by the analysis of decay time, rise time and slope of the rising phase (Fig. $4 D-F$ ).

Synapsin I deletion reduces the size of the readily releasable pool, but not the release probability or the quantal size of GABA transmission

We next investigated how the lack of SynI affects the elementary events responsible for GABA transmission leading to a decreased eIPSC size. To this aim, we used multiple probability fluctuation analysis (MPFA) (Clements and Silver, 2000; Baldelli et al., 2005), which provides direct information on the average quantal $P r_{\mathrm{av}}$, the number of independent release sites $\left(N_{\min }\right)$ and the average postsynaptic quantal size $\left(Q_{\mathrm{av}}\right)$. This crucial information was derived from the relationship between variance $\left(\sigma^{2}\right)$ and mean amplitude of eIPSCs $\left(I_{\text {av }}\right)$ recorded under various $\operatorname{Pr}$ conditions. We changed $\operatorname{Pr}$ by adding increasing concentrations of $\mathrm{Cd}^{2+}(0,2,4$, and $6 \mu \mathrm{M})$ to the extracellular medium (Fig. 5 A). Variancemean plots were fitted with a simple parabola (Fig. $5 B$ ) yielding direct estimates of $N_{\min }$ and $Q_{a v}$, whereas $P r_{\mathrm{av}}$ was calculated from $P r_{\mathrm{av}}=I_{\mathrm{av}} / N_{\mathrm{min}} Q_{\mathrm{av}} \cdot Q_{\mathrm{av}}$ was derived from the initial slope of the parabola and corrected for the factor $1 / 1+\left(C V_{i}^{2}\right)$, with $C V_{i}$ indicating the coefficient of variation of mIPSCs at an individual site (see Materials and Methods).

The mean eIPSC amplitude was $~ 30 \%$ smaller in $\mathrm{KO}$ neurons $[0.84 \pm 0.73$ and $0.6 \pm 0.4 \mathrm{nA}$ in WT $(n=9)$ and $\mathrm{KO}(n=$ 8) neurons, respectively; $p<0.02$ ] (Fig. 5C). The analysis revealed a similar $30 \%$ decrease of $N_{\min }$ in SynI KO neurons $(193 \pm 12$ and $140 \pm 9$ in WT and KO neurons, respectively; $p<0.01$ ) (Fig. $5 D$ ), whereas no differences were found in the mean $P r_{\mathrm{av}}(0.53 \pm 0.03$ and $0.51 \pm 0.03$ in $\mathrm{WT}$ and $\mathrm{KO}$ neurons, respectively; $p=$ 0.73 ) (Fig. $5 E$ ) and $Q_{\mathrm{av}}(8.3 \pm 0.32$ and $8.4 \pm 0.33 \mathrm{pA}$ in WT and $\mathrm{KO}$ neurons, respectively; $p=0.74$ ) (Fig. $5 F$ ) between WT and KO neurons. It is noteworthy that MPFA slightly underestimated the mean $Q_{\mathrm{av}}$ with respect to the mean mIPSCs (12.26 in KO vs 12.46 in WT), because of various causes that equally influence the recordings of WT and $\mathrm{KO}$ neurons (Baldelli et al., 2005).

To further evaluate the effects of SynI deletion on the $P r$ and size of the RRP, we used an alternative method which analyzes the cumulative amplitude profile during high-frequency trains of stimuli ( $1 \mathrm{~s}$ at 40 $\mathrm{Hz}$ ) (Schneggenburger et al., 1999; Baldelli et al., 2005). As shown in Figure 6 A, a significant depression of eIPSCs became apparent during the trains in both WT and SynI $\mathrm{KO}$ neurons. In both experimental groups, the cumulative profile of repeated eIPSCs showed a rapid rise followed by a slower linear increase of different steepness at later pulses (Fig. 6B). Assuming that the slow linear rise is attributable to the equilibrium between the release-induced depletion and the constant replenishment of the RRP, back-extrapolation of the linear portion to time 0 yields a rough estimation of the total release minus the total replenishment, corresponding to the size of the RRP of synchronous release $\left(\mathrm{RRP}_{\text {syn }}\right)$ (Schneggenburger et al., 1999). As shown in Figure $6 C$, the $\mathrm{RRP}_{\text {syn }}$ was significantly decreased in $\mathrm{KO}$ neurons $(1320 \pm 59$ and $966 \pm 53 \mathrm{pA}$ in WT and KO neurons, respectively; $n=25$ for both genotypes; $p<0.01)$ to the same extent of the mean amplitude of the first IPSC in the train $(791 \pm 36$ and $569 \pm 32 \mathrm{pA}$ in WT and KO neurons, respectively; $p<0.01$ ) (Fig. $6 D)$. Synaptic vesicle release probability, $P_{\text {ves }}$, calculated as the ratio between the first IPSC $\left(I_{1}\right)$ and $\mathrm{RRP}_{\text {syn }}$ (see Materials and Methods) was not significantly affected $(0.6 \pm 0.02$ and $0.6 \pm$ 0.03 in WT and KO neurons, respectively; $p=0.88$ ) (Fig. $6 E$ ). To estimate the number of $S V s\left(N_{\text {syn }}\right)$ forming the RRP $\mathrm{Ryn}_{\text {syn }}$, we divided $R R P_{\text {syn }}$ by $Q_{a v}$ (i.e., by the average amplitude of mIPSCs obtained by MPFA) (see above). $N_{\text {syn }}$ was found to be decreased by SynI deletion $(160 \pm 8$ and $114 \pm 6$ in WT and 
KO neurons, respectively; $p<0.01$ ), yielding a number of RRP vesicles lower than, but comparable with, the number of active sites independently evaluated by MPFA.

The $\mathrm{RRP}_{\text {total }}$ and the $\operatorname{Pr}_{\text {total }}$ were also estimated by using hypertonic stimulation (Rosenmund and Stevens, 1996). Voltage-clamped autaptic hippocampal neurons were initially stimulated at low frequency $(0.05 \mathrm{~Hz})$ to evoke single eIPSCs. One minute later, a hypertonic solution (normal extracellular solution supplemented with $500 \mathrm{~mm}$ sucrose) (Yamaguchi et al. 2002) was focally microapplied to the same autaptic neuron (Fig. 7A). Consistent with the data reported above, the charge transfer in the transient part of the eIPSC showed a $\sim 30 \%$ decrease in $\mathrm{KO}$ neurons [87.7 \pm 6.8 and $65.6 \pm 5.0 \mathrm{pC}$, for WT $(n=8)$ and $\mathrm{KO}(n=8)$ neurons, respectively; $p<0.05$ ] (Fig. $7 B, C$ ). The charge transfer in the transient part of the highsucrose induced synaptic current, a reliable measure of $\mathrm{RRP}_{\text {total }}$ (Rosenmund and Stevens, 1996), revealed a significant decrease in KO neurons $(167 \pm 14$ and $131 \pm 8 \mathrm{pC}$ in $\mathrm{WT}$ and $\mathrm{KO}$ neurons, respectively; $p<0.05$ ) (Fig. $7 B, D$ ). However, no difference was detected between the two genotypes in the $P r_{\text {total }}$ of the first EPSC, estimated by dividing the charge transfer of the first eIPSC by the $\mathrm{RRP}_{\text {total }}($ Fig. $7 E$ ).

\section{Synapsin I deletion preserves the} expression level of presynaptic proteins and the density of GABAergic synaptic contacts

The decreased RRP size observed in the absence of synapsin by double patchclamp recordings of monosynaptically connected neurons could be ascribed to either a lower number of synaptic contacts between the two neurons or a decrease in the number of SVs released per inhibitory synapse, or both. Given that SynI deletion has been reported to delay synaptogenesis (Chin et al., 1995) (for review, see Ferreira and Rapoport, 2002), we performed a biochemical and immunocytochemical analysis to verify whether the observed decrease in the RRP size could result from a decrease in the number of GABAergic synaptic contacts between couples of presynaptic and postsynaptic neurons. To test this hypothesis we evaluated the expression levels of presynaptic and GABAergic markers and compared the density of inhibitory terminals in WT and KO neurons over the entire culture period (4-21 DIV).

We first estimated the expression level of presynaptic proteins including synapsins I/II and synaptophysin as well as of specific markers of inhibitory neurons such as the GABA synthesizing enzyme GAD67. As shown in Figure $8 A$, the expression of the various markers increases, as expected, over development in vitro. However, no difference was observed between
WT and KO hippocampal neurons in the expression level of any of the analyzed markers (Fig. 8 B). Because SynI deletion could affect the specific targeting of presynaptic proteins also in the presence of similar expression levels in the total cell lysate, we visualized inhibitory nerve terminals by V-GAT immunofluorescence during in vitro development (Fig. 8C). V-GAT-positive puncta strictly colocalized with synaptophysin were considered inhibitory terminals and their total number was automatically counted, as described in Materials and Methods. The number of V-GAT-positive nerve terminals largely increased with time in culture in both WT and $\mathrm{KO}$ neurons in a similar manner (Fig. $8 D$ ). Because patch-clamp recordings predominantly analyze axosomatic synapses, counting of somatic GABAergic synapses was also performed as described in Materials and Methods at 21 DIV. In line with the findings reported above, the number of somatic inhibitory terminals was not significantly different between WT and KO cultures $\left(0.058 \pm 0.007\right.$ and $0.059 \pm 0.029$ synapses $/ \mu \mathrm{m}^{2}$ for 

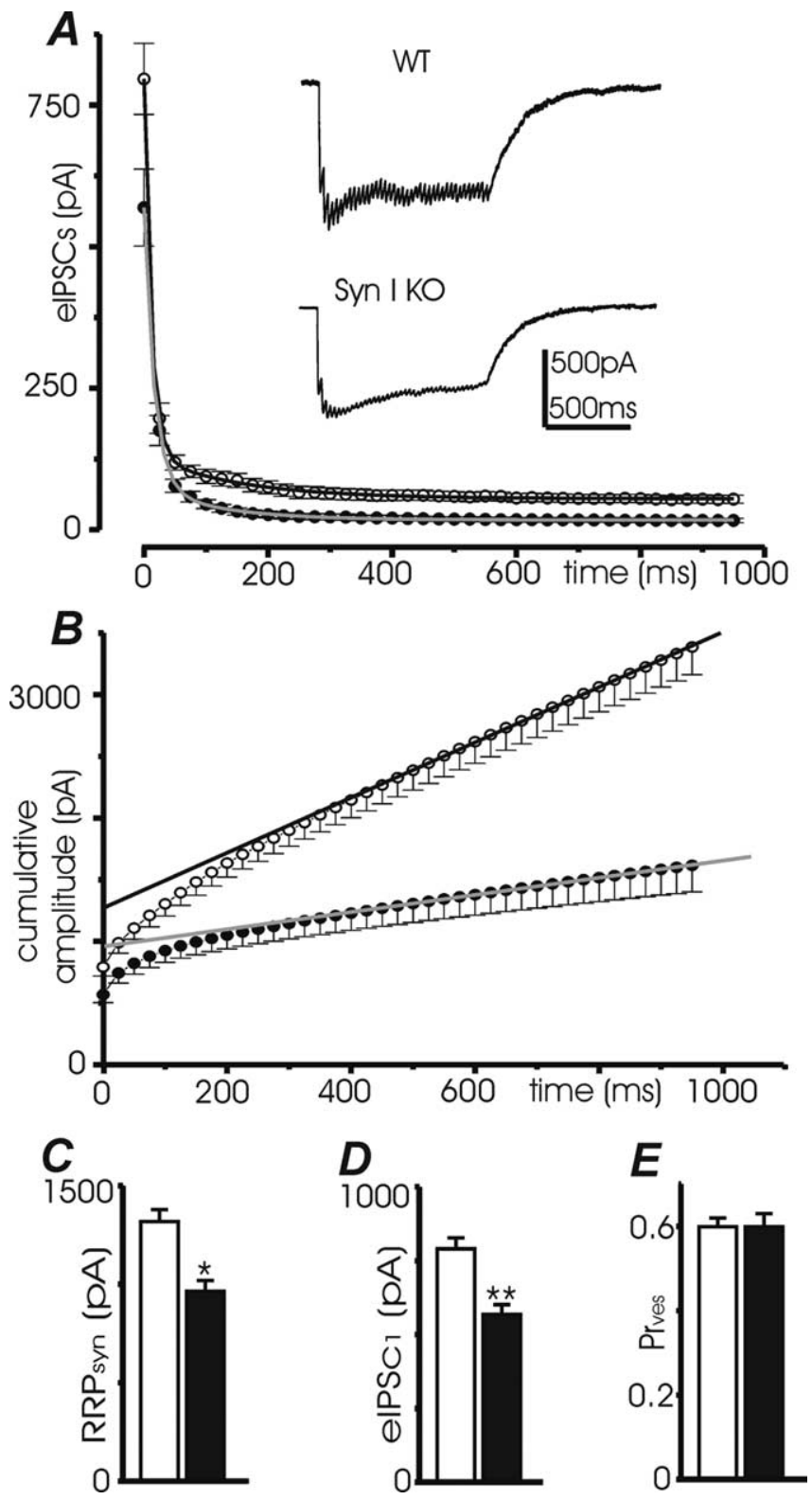

Figure 6. Estimate of RRP and Pr by using the cumulative amplitude profile analysis. A, Plot of mean elPSCs amplitude ( \pm SE) versus time during repetitive stimulations at $40 \mathrm{~Hz}$ of WT and Synl KO neurons (21-28 DIV; $n=25$ for both genotypes) fitted with the biexponential function: $I(t)=y_{o}+A_{f} e^{(-t / \tau f)}+A_{s} e^{(-t / \tau s)}$ (WT, black trace; KO, gray trace). Representative recordings during a train of 40 stimuli at $40 \mathrm{~Hz}$ in single WT and Synl KO neurons are shown in the inset. Extracellular stimulation artifacts were removed. $\boldsymbol{B}$, Cumulative elPSC amplitude profile. To estimate the RRP, data points in the range of $0.4-0.9 \mathrm{~s}$ were fitted by linear regression and back-extrapolated to time 0 (WT, black trace; KO, gray trace) to estimate the cumulative elPSC amplitude before steady-state depression $\left(\operatorname{RRP}_{\text {syn }}\right) \cdot \mathbf{C}-\boldsymbol{E}$, The size of $\operatorname{RRP}_{\text {syn }}(\boldsymbol{C})$, the amplitude of the first elPSC in the train $(\boldsymbol{D})$, and the calculated $P r_{\text {ves }}(\boldsymbol{E})$ are shown as means \pm SE (WT, white bars; $\mathrm{KO}$, black bars). ${ }^{* *} p<0.01$ versus WT, two-tailed Student's t test. $n=25$ for both WT and Synl KO.

WT and KO cultures, respectively; $n=10-15$ fields from three independent culture preparations per genotype). These findings demonstrate that lack of SynI does not significantly affect the number of GABAergic contacts in mature cultures and suggest that the impairment in IPSC amplitude is caused by a decrease in the number of SVs released by the action potential at the level of a single synapse.
Synapsin I deletion decreases the number of SVs released in response to depolarization of single GABAergic terminals To ascertain whether SynI deletion can indeed induce a decrease in the number of SVs released by the action potential at the level of the single presynaptic terminal, as suggested by the abovereported electrophysiological and immunocytochemical data, we examined putative single GABAergic contacts in low-density hippocampal cultures. Physiologically active axon terminals were identified using the styryl dye FM1-43 which is taken up in an activity-dependent manner at sites of active SV exoendocytosis (Kirischuk et al., 1999) (Fig. 9A). Evoked IPSCs were elicited in the presence of a $\mathrm{Na}^{+}$-channel blocker by direct depolarization of single FM1-43-stained boutons (Chen et al., 2004). This method has the advantage that the range of presynaptic $\left[\mathrm{Ca}^{2+}\right]_{\text {in }}$ could be tuned by precise gradation of the presynaptic depolarization (Kirischuk et al., 1999). Preliminary experiments were performed to set the intensity and duration of the stimulation that minimized the number of failures and obtained the highest mean eIPSC amplitude (Fedulova et al., 1999). We used constant stimulation intensity and duration ( $1 \mathrm{~ms}$ at $5 \mu \mathrm{A})$ to evoke eIPSCs at WT and KO neuron synapses. Similarly to what observed with monosynaptic eIPSCs (Fig. 1), single-synapse eIPSCs had a reduced amplitude in $\mathrm{KO}$ neurons with respect to WT neurons (Fig. 9B). The analysis of the mean probability density (Fig. 9C) resolved a multiquantal distribution, with a first peak related to the unitary quantum centered at $12 \mathrm{pA}$ for both WT and $\mathrm{KO}$ neurons, in good agreement with the value obtained by mIPSC analysis (Fig. 4). It is interesting to note that single SV release was a less probable event, although multiquantal release was more frequent, as a consequence of the increase in $\operatorname{Pr}$ because of the high intensity of the extracellular stimulation (Kirischuk et al., 2002). The mean probability density histogram showed a clear redistribution of the probability of events toward lower numbers of quanta in SynI KO neurons, consistent with the observed decrease in the number of SVs in the RRP (see Figs. 5-7). Despite these changes, similar failure rates were found in WT and $\mathrm{KO}$ neurons $(12.95 \pm 0.72$ and $13.93 \pm 0.51 \%$ for WT and SynI KO neurons, respectively; $p=0.26$ ), probably because of the high release probability reached with the strong extracellular depolarization and/or the contamination by stimulation failures. Consistent with the redistribution of the probability of quantal events, the shape of the cumulative frequency versus amplitude curve was significantly steeper in SynI KO neurons (Fig. 9D) and the mean value of single eIPSC amplitude was significantly decreased $[37.02 \pm 2.6$ and $28.3 \pm 2.3 \mathrm{pA}$ for WT $(n=9)$ and SynI KO $(n=11)$ neurons, respectively; $p<0.01$ ] (Fig. 9E). Given an average amplitude of $\sim 12 \mathrm{pA}$ for the unitary quantum in both WT and SynI KO neurons (see above) (Fig. 4), the single-synapse eIPSC was three times larger in WT mice and only two times larger in $\mathrm{KO}$ mice. This suggests that an isolated action potential is able to synchronously release, on average, three SVs from single WT GABAergic terminals, and only two SVs from SynI-deficient GABAergic terminals.

\section{Discussion}

Although a large number of SV genes encoding for SV proteins (Takamori et al. 2006) have been inactivated in animal models to uncover the physiological role of the respective proteins, only very few mutants have been reported to exhibit an epileptic phenotype (Noebels, 2003). A severe epileptic phenotype was found in genetically altered mice lacking members of the Syn (for review, see Baldelli et al., 2006) and SV2 (Crowder et al., 1999; Janz et al., 1999; Custer et al., 2006) families, whereas epilepsy was not 
observed in mouse mutants deleted for other SV or presynaptic plasma membrane proteins. Interestingly, a new form of familial X-linked epilepsy characterized by a nonsense mutation in the SYN1 gene was recently reported (Garcia et al., 2004), making SynI KO mice the only experimental model of human epilepsy associated with SV defects. Epileptic seizures are generated by an initially localized hyperexcitability that spreads into a series of interconnected neuronal networks, where it might not be properly counterbalanced and circumscribed by inhibitory mechanisms (Steinlein, 2004). Thus, the appearance of an epileptic phenotype in mice and man associated with mutations of the SYN1 gene implicates SynI in the delicate balance between inhibitory and excitatory transmission that controls cortical excitability and indicates that the increased seizure propensity of SynI mutants can be ascribed to specific roles of SynI in synaptic transmission.

Syn isoforms are present in virtually all conventional chemical synapses, although the expression levels and the isoform spectrum differs (Sudhof et al., 1989; Kielland et al., 2006; Bragina et al., 2007), thus contributing to the heterogeneity in the SV protein complement and in the functional properties of the synapses (Atwood and Karunanithi, 2002). Consistent with the major role of the Syns in the assembly of the RP of SVs through SV clustering and actin binding (Benfenati et al., 1992, 1993; Ceccaldi et al., 1995), central synapses in mice lacking one or more Syn isoforms (with the notable exception of SynIII KO mice) display a marked decrease in SV density, reflecting a depletion of the RP (Li et al., 1995; Rosahl et al., 1995, Takei et al., 1995; Gitler at al., 2004; Siksou et al., 2007). In addition, SynI KO neurons suffer of a reduced size of the recycling pool and a decreased recruitment of recycling SVs to the RRP during synapse maturation (Ryan et al., 1996; Mozhayeva et al., 2002). These changes seem to occur to a larger extent at excitatory than at inhibitory synapses (Terada et al., 1999; Gitler et al., 2004). If the epileptic phenotype is the expression of an unbalance between excitatory and inhibitory circuits, it is fundamental to evaluate whether SynI could affect in a different way the physiology of excitatory and inhibitory synapses. Although multiple defects in short-term plasticity paradigms have been described at excitatory synapses (Rosahl et al., 1995; Gitler et al., 2004; Sun et al., 2006), a thorough characterization of the effects of SynI deletion on inhibitory synapses was missing, despite the reduction in IPSC amplitude in response to single stimuli found in SynI, SynIII, and SynI/II/III KO neurons (Terada et al., 1999; Feng et al., 2002; Gitler et al., 2004), which was never observed in excitatory synapses.

In this study, we performed a detailed characterization of the impairment in GABA quantal release mechanisms and shortterm plasticity at hippocampal inhibitory neurons. We found that GABA release is impaired in response to various stimulation protocols, from single pulses to high-frequency trains. The de8 for both genotypes.
B
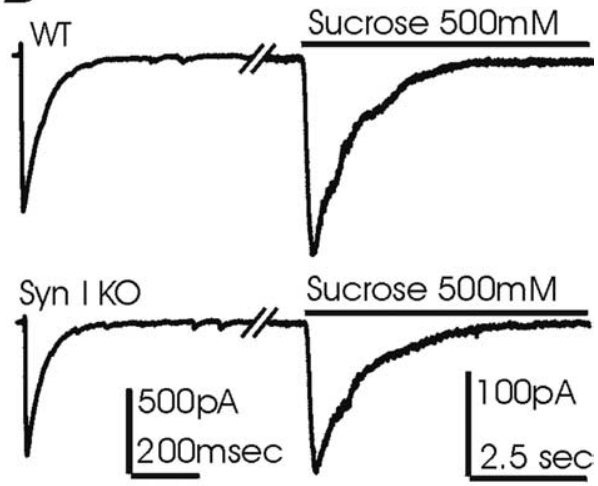

D E
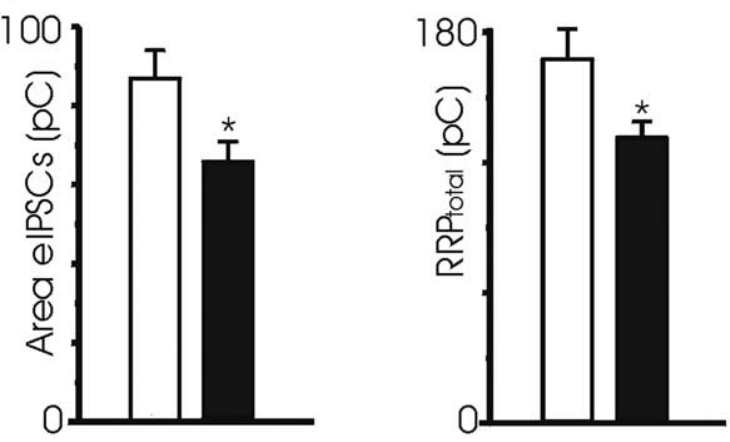

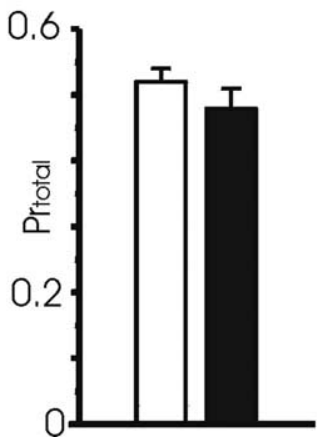

Figure 7. Estimation of $\mathrm{RRP}_{\text {total }}$ and $P r_{\text {total }}$ by hypertonic stimulation of autaptic GABAergic neurons. $\boldsymbol{A}$, Phase-contrast micro-

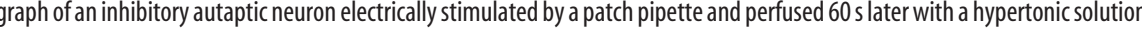
of the response induced by the hypertonic solution. (WT, white bars; K0, black bars) ${ }^{*} p<0.05$, two-tailed Student's $t$ test. $n=$

creased eIPSC amplitude in response to single pulses observed in SynI KO neurons was found to depend entirely on a parallel decrease in the size of the RRP, although the quantum size and the probability of release were unaffected. This finding confirms a role of SynI in the membrane events of exocytosis, as suggested by previous data (Hilfiker et al., 1999, 2005; Humeau et al., 2001; Fassio et al., 2006; Hvalby et al., 2006; Sun et al., 2006). Consistently, forms of presynaptic plasticity that rely on a change in release probability boosted by $\left[\mathrm{Ca}^{2+}\right]_{\mathrm{i}}$ build-up, such as pairedpulse stimulation (Zucker and Regehr, 2002) are not affected by the SynI deletion.

The significant decrease in GABA release during highfrequency stimulation and the slower recovery from depression observed in $\mathrm{KO}$ neurons indicate the existence of deficits in the SV recycling mechanisms and/or in the recruitment of SVs from the RP. The importance of SynI in depression of GABA release strongly depended on the rate of presynaptic stimulation. Whereas at low stimulation frequencies WT and KO terminals exhibited a similar synaptic strength, at higher stimulation frequencies synaptic depression was more severe and recovery from depression was slower in SynI KO terminals. Thus, SynI appears to be essential for enabling neurons to cope with high-frequency activity, by boosting synaptic responses during the depression plateau phase and accelerating recovery from depression to the basal state.

The effect of SynI deletion on both depression and recovery in 
$A$

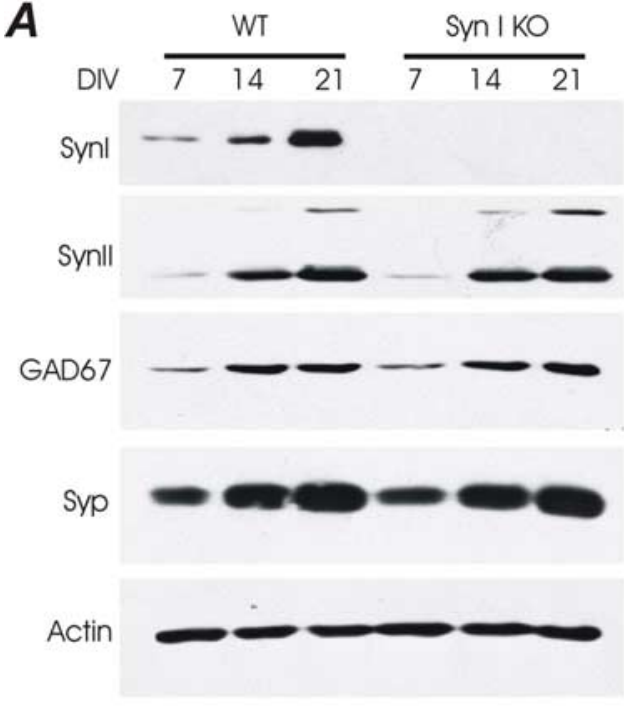

C

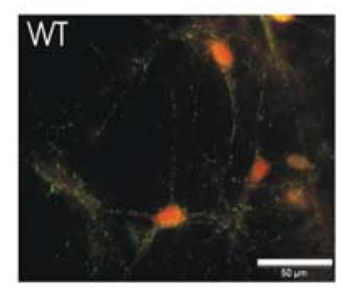

$B$

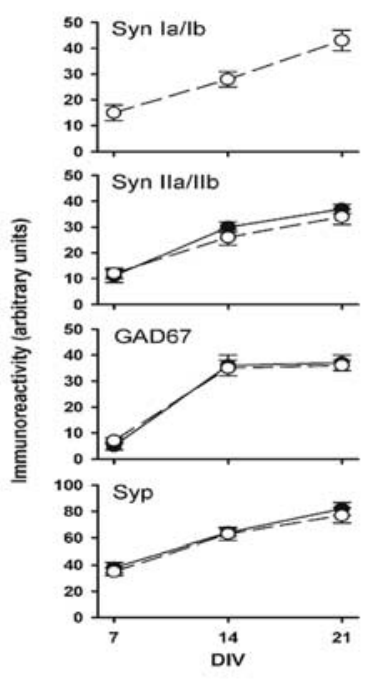

$D$

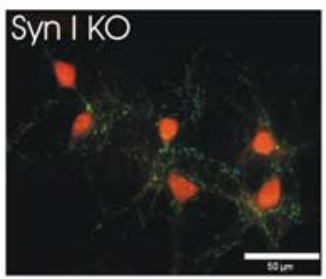

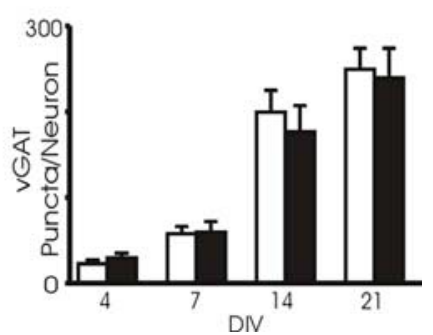

Figure 8. Expression of presynaptic proteins and number of GABAergic synaptic contacts are not affected by Synl deletion in hippocampal neurons during in vitro differentiation. $A$, Protein extracts ( $10 \mu \mathrm{g} / \mathrm{lane})$ from WT and Synl KO hippocampal neurons were prepared at the indicated DIV and analyzed for the expression levels of synapsin la/lb (Synl), synapsin Ila/Ilb (Synll), GAD67, and synaptophysin (Syp). A representative immunoblot is shown. Equal loading was confirmed by the constant actin levels. $\boldsymbol{B}$, Immunoblots from three independent replications of the experiment shown in A were quantitatively analyzed by densitometric scanning of the fluorograms. The developmental patterns of expression of Synl, Synll, GAD67 and Syp in WT (open symbols) and KO (closed symbols) neurons as a function of the days in vitro (DIV) are plotted as means \pm SE in arbitrary units. C, Hippocampal neurons from WT (left panel) or Syn I KO (right panel) mice were fixed at 21 DIV and immunostained for V-GAT (green) and NeuN (red). V-GAT-positive puncta were counted and normalized by the cell number. Scale bar: $50 \mu \mathrm{m}$. $\boldsymbol{D}$, The total number of inhibitory terminals in WT (white bars) and Synl KO (black bars) cultures was obtained by automatic counting and divided by the number of neurons in the field ( $10-15$ images for each age in culture and from at least 3 preparations conducted in parallel). No significant differences in the density of GABAergic terminals were observed between WT and KO neurons at the various DIV analyzed.

GABAergic neurons was occluded by preventing the increase in intraterminal $\left[\mathrm{Ca}^{2+}\right]_{\mathrm{i}}$ by intracellular EGTA. This effect, which was reported previously to occur also in excitatory synapses (Sun et al., 2006), strongly suggests that use-dependent synaptic plasticity during sustained high-frequency stimulation is regulated by a progressive increase in intraterminal $\mathrm{Ca}^{2+}$ activating $\mathrm{Ca}^{2+} /$ calmodulin-dependent and adenylate cyclase/cAMP-dependent phosphorylation pathways impinging on the Syns and promoting recruitment of reserve SVs to the RRP (Benfenati et al., 1992; Ceccaldi et al., 1995; Hosaka et al., 1999; Chi et al. 2001, 2003, Menegon et al., 2006). Such a mechanism was completely missing in neurons lacking SynI, which displayed a behavior superimposable to that of WT neurons treated with EGTA. This finding also indicates that, despite the fact that all Syn isoforms can be phosphorylated by the above-mentioned signal transduction pathways, SynI is the key isoform that plays a role in the adaptation of nerve terminals during use-dependent depression. The lack of change in use-dependent depression found in SynI/II/III KO

inhibitory neurons (Gitler et al., 2004) further indicates that the three Syn gene products may have distinct and nonredundant functions in nerve terminal physiology.

GABA is physiologically important both during neural development and in adult brain. Thus, it is possible that the epileptic phenotype is caused by either an early disruption of neuronal development resulting from the impaired trophic action of excitatory GABA transmission (Represa and Ben-Ari, 2005; Cancedda et al., 2007) or a lack of physiological inhibition in mature neuronal networks. Our data demonstrate that the impairment in GABA transmission is developmentally regulated and appears only at late stages of synaptic maturation ( $>15-21$ DIV), strongly suggesting that that seizure propensity of SynI KO mutants is attributable to an abnormal regulation of excitability that induces an anomalous spread of excitation along the neuronal networks.

Interestingly, some of the effects of the deletion of the only other SV protein which causes epilepsy, namely SV2A, resemble those observed in SynI KO inhibitory neurons, suggesting possible common mechanisms in the pathogenesis of the epileptic phenotype. Stimulus-evoked neurotransmitter release is reduced in hippocampal neurons as well as chromaffin cells lacking SV2A, indicating that SV2A plays a crucial role in the maintenance of a RRP of fusioncompetent SV (Crowder et al., 1999; Xu and Bajjalieh, 2001; Custer et al., 2006). The decrease in the RRP observed after SV2A deletion $(\sim 40 \%)$ is of the same order of magnitude of that observed in hippocampal GABAergic neurons of SynI KO mice $(\sim 30 \%)$. The decrease in the RRP size and the absence of changes in the release probability observed in SynI and SV2A KO

neurons suggest that both SynI and SV2A physiologically sustain low-frequency neurotransmission by selectively enhancing priming of SVs and regulating RRP size.

A question that remains open is how to explain the distinct physiological phenotype of SynI deletion at glutamatergic and GABAergic neurons leading to network hyperexcitability, given the widespread distribution of Syns in both types of neurons. Synapses are functionally heterogeneous in nature and synaptic diversification depends mainly on the type of activity (phasic vs tonic), different sizes of SV pools and differential distribution of synaptic proteins (Atwood and Karunanithi, 2002). In SynI KO neurons, glutamatergic transmission is impaired during sustained high-frequency activity, but is fully preserved in response to single action potentials. However, GABAergic transmission is defective during both basal electrical activity and high-frequency stimulation. Under this condition, both the phasic synaptic inhibition and the tonic extrasynaptic inhibition caused by GABA 
spillover are decreased. This impairment of the inhibitory mechanisms may force neuronal circuits into a state of basal heightened excitability which facilitates the spontaneous or stimulus-evoked onset of epileptic seizures.

\section{References}

Atwood HL, Karunanithi S (2002) Diversification of synaptic strength: presynaptic elements. Nat Rev Neurosci 3:497-516.

Baldelli P, Forni PE, Carbone E (2000) BDNF, NT-3 and NGF induce distinct new $\mathrm{Ca}^{2+}$ channel synthesis in developing hippocampal neurons. Eur J Neurosci 12:4017-4032.

Baldelli P, Novara M, Carabelli V, HernándezGuijo JM, Carbone E (2002) BDNF upregulates evoked GABAergic transmission in developing hippocampal neurons by potentiating presynaptic $\mathrm{N}-$ and $\mathrm{P} / \mathrm{Q}$-type $\mathrm{Ca}^{2+}$ channels signaling. Eur J Neurosci 16:2297-2310.

Baldelli P, Hernandez-Guijo JM, Carabelli V, Carbone E (2005) Brain-derived neurotrophic factor enhances GABA release probability and nonuniform distribution of $\mathrm{N}$ - and $\mathrm{P} / \mathrm{Q}-$ type channels on release sites of hippocampal inhibitory synapses. J Neurosci 25:3358-3368.

Baldelli P, Fassio A, Corradi A, Valtorta F, Benfenati $\mathrm{F}$ (2006) The synapsins and the control of neuroexocytosis. In: Molecular mechanisms of exocytosis (Regazzi R, ed), pp 62-74. Georgetown, TX: Landes Bioscence.

Barbara J-G, Takeda K (1996) Quantal release at a neuronal nicotinic synapse from rat adrenal gland. Proc Natl Acad Sci USA 93:9905-9909.

Bekkers JM, Stevens CF (1991) Excitatory and inhibitory autaptic currents in isolated hippocampal neurons maintained in cell culture. Proc Natl Acad Sci USA 88:7834-7838.

Benfenati F, Valtorta F, Chieregatti E, Greengard P (1992) Interaction of free and synaptic vesicle-bound synapsin I with F-actin. Neuron $8: 377-386$.

Benfenati F, Valtorta F, Rossi MC, Onofri F, Sihra T, Greengard P (1993) Interactions of synapsin I with phospholipids: possible role in synaptic vesicle clustering and in the maintenance of bilayer structures. J Cell Biol 123:1845-1855.

Bonanomi D, Menegon A, Miccio A, Ferrari G, Corradi A, Kao HT, Benfenati F, Valtorta F (2005) Phosphorylation of synapsin I by cAMP-dependent protein kinase controls synaptic vesicle dynamics in developing neurons. J Neurosci 25:7299-7308.

Bragina L, Candiracci C, Barbaresi P, Giovedi S, Benfenati F, Conti F (2007) Heterogeneity of glutamatergic and GABAergic release machinery in cerebral cortex. Neuroscience 146:1829-1840.

Cancedda L, Fiumelli H, Chen K, Poo M-m (2007) Excitatory GABA action is essential for morphological maturation of cortical neurons in vivo. J Neurosci 27:5224-5235.

Ceccaldi PE, Grohovaz F, Benfenati F, Chieregatti E, Greengard P, Valtorta F (1995) Dephosphorylated synapsin I anchors synaptic vesicles to actin cytoskeleton: an analysis by videomicroscopy. J Cell Biol 128:905-912.

Chen G, Harata NC, Tsien RW (2004) Paired-pulse depression of unitary quantal amplitude at single hippocampal synapses. Proc Natl Acad Sci USA 101:1063-1068.

Chi P, Greengard P, Ryan TA (2001) Synapsin dispersion and reclustering during synaptic activity. Nat Neurosci 4:1187-1193.

Chi P, Greengard P, Ryan TA (2003) Synaptic vesicle mobilization is regulated by distinct synapsin I phosphorylation pathways at different frequencies. Neuron 38:69-78.

Chin LS, Li L, Ferreira A, Kosik KS, Greengard P (1995) Impairment of axonal development and of synaptogenesis in hippocampal neurons of synapsin I-deficient mice. Proc Natl Acad Sci USA 92:9230-9234.
$B$
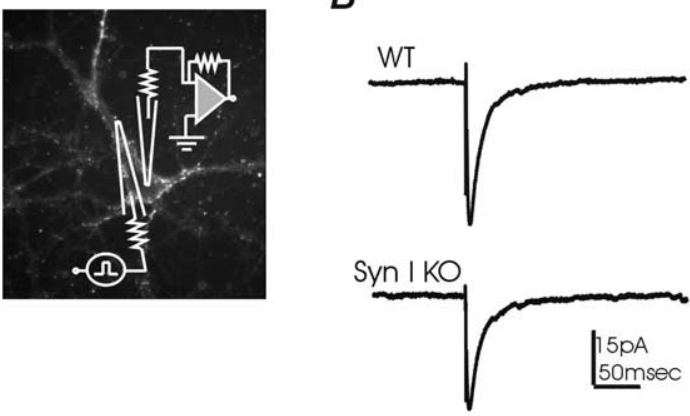

$D$

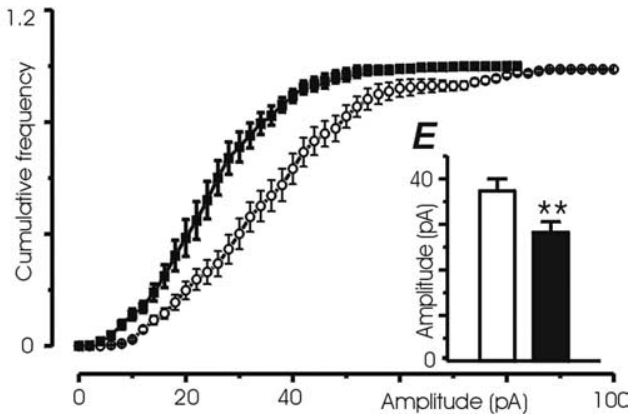

gure 9. Analysis of GABAergic transmission at single synaptic boutons. $\boldsymbol{A}$, Left, Phase-contrast micrograph of a glass pipette ion ( $f$ ) and a recording patch pipette ( $r$ ) on a hippocampal neuron. Right, Fluorescence image showing presyn$2 \mathrm{pA}$ ). $\boldsymbol{E}$, In the inset, the mean amplitude ( $\pm \mathrm{SE}$ ) of elPSC is reported for WT (white bar) and Synl KO (black bar) neurons. ${ }^{* *} p<$ 0.01 versus WT, two-tailed Student's $t$ test.

Clements JD, Silver RA (2000) Unveiling synaptic plasticity: a new graphical and analytical approach. Trends Neurosci 23:105-113.

Crowder KM, Gunther JM, Jones TA, Hale BD, Zhang HZ, Peterson MR, Scheller RH, Chavkin C, Bajjalieh SM (1999) Abnormal neurotransmission in mice lacking synaptic vesicle protein 2A (SV2A). Proc Natl Acad Sci USA 96:15268-15273.

Custer KL, Austin NS, Sullivan JM, Bajjalieh SM (2006) Synaptic vesicle protein 2 enhances release probability at quiescent synapses. J Neurosci 26:1303-1313.

Fassio A, Merlo D, Mapelli J, Menegon A, Corradi A, Mete M, Zappettini S, Bonanno G, Valtorta F, D’Angelo E, Benfenati F (2006) The synapsin domain $\mathrm{E}$ accelerates the exoendocytotic cycle of synaptic vesicles in cerebellar Purkinje cells. J. Cell Sci 119:4257-4268.

Fedulova SA, Vasilyev EV, Isaeva EV, Romanyuk SG, Veselovsky NS (1999) Possibility of multiquantal release at single inhibitory synapse in cultured rat hippocampal neurons. Neuroscience 92:1217-1230.

Feng J, Chi P, Blanpied TA, Xu Y, Magarinos AM, Ferreira A, Takahashi RH, Kao HT, McEwen BS, Ryan TA, Augustine GJ, Greengard P (2002) Regulation of neurotransmitter release by synapsin III. J Neurosci 22:4372-4380

Ferreira A, Rapoport M (2002) The synapsins: beyond the regulation of neurotransmitter release. Cell Mol Life Sci 59:589-595.

Ferreira A, Kao HT, Feng J, Rapoport M, Greengard P (2000) Synapsin III: developmental expression, subcellular localization, and role in axon formation. J Neurosci 20:3736-3744.

Garcia CC, Blair HJ, Seager M, Coulthard A, Tennant S, Buddles M, Curtis A, Goodship JA (2004) Identification of a mutation in synapsin I, a synaptic vesicle protein, in a family with epilepsy. J Med Genet 41:183-186.

Gitler D, Takagishi Y, Feng J, Ren Y, Rodriguiz RM, Wetsel WC, Greengard P, Augustine GJ (2004) Different presynaptic roles of synapsins at excitatory and inhibitory synapses. J Neurosci 24:11368-11380.

Hilfiker S, Schweizer FE, Kao HT, Czernik AJ, Greengard P, Augustine GJ 
(1998) Two sites of action for synapsin domain $\mathrm{E}$ in regulating neurotransmitter release. Nat Neurosci 1:29-35.

Hilfiker S, Pieribone VA, Czernik AJ, Kao HT, Augustine GJ, Greengard P (1999) Synapsins as regulators of neurotransmitter release. Philos Trans R Soc Lond B Biol Sci 354:269-279.

Hilfiker S, Benfenati F, Doussau F, Nairn AC, Czernik AJ, Augustine GJ, Greengard P (2005) Structural domains involved in the regulation of transmitter release by synapsins. J Neurosci 25:2658-2669.

Hosaka M, Hammer RE, Sudhof TC (1999) A phospho-switch controls the dynamic association of synapsins with synaptic vesicles. Neuron 24:377-387.

Humeau Y, Doussau F, Vitiello F, Greengard P, Benfenati F, Poulain B (2001) Synapsin controls both reserve and releasable synaptic vesicle pools during neuronal activity and short-term plasticity in Aplysia. J Neurosci 21:4195-4206.

Hvalby O, Jensen V, Kao HT, Walaas SI (2006) Synapsin-regulated synaptic transmission from readily releasable synaptic vesicles in excitatory hippocampal synapses in mice. J Physiol (Lond) 571:75-82.

Janz R, Goda Y, Geppert M, Missler M, Sudhof TC (1999) SV2A and SV2B function as redundant $\mathrm{Ca}^{2+}$ regulators in neurotransmitter release. Neuron 24:1003-1016.

Jensen K, Jensen MS, Lambert JD (1999) Post-tetanic potentiation of GABAergic IPSCs in cultured rat hippocampal neurones. J Physiol (Lond) 519:71-84.

Kielland A, Erisir A, Walaas SI, Heggelund P (2006) Synapsin utilization differs among functional classes of synapses on thalamocortical cells. J Neurosci 26:5786-5793.

Kirischuk S, Veselovsky N, Grantyn R (1999) Relationship between presynaptic calcium transients and postsynaptic currents at single gammaaminobutyric acid (GABA)ergic boutons. Proc Natl Acad Sci USA 96:7520-7525.

Kirischuk S, Clements JD, Grantyn R (2002) Presynaptic and postsynaptic mechanisms underlie paired pulse depression at single GABAergic boutons in rat collicular cultures. J Physiol (Lond) 543:99-116.

Li L, Chin LS, Shupliakov O, Brodin L, Sihra TS, Hvalby O, Jensen V, Zheng D, McNamara JO, Greengard P, Andersen P (1995) Impairment of synaptic vesicle clustering and of synaptic transmission, and increased seizure propensity, in synapsin I-deficient mice. Proc Natl Acad Sci USA 92:9235-9239.

Mallart A, Martin AR (1967) An analysis of facilitation of transmitter release at the neuromuscular junction of the frog. J Physiol (Lond) 193:679-694.

Menegon A, Bonanomi D, Albertinazzi C, Lotti F, Ferrari G, Kao HT, Benfenati F, Baldelli P, Valtorta F (2006) Protein kinase A-mediated synapsin I phosphorylation is a central modulator of $\mathrm{Ca}^{2+}$-dependent synaptic activity. J Neurosci 26:11670-11681.

Mozhayeva MG, Sara Y, Liu X, Kavalali ET (2002) Development of vesicle pools during maturation of hippocampal synapses. J Neurosci 22:654-665.

Noebels JL (2003) The biology of epilepsy genes. Annu Rev Neurosci 26:599-625.

Reid CA, Clements JD (1999) Postsynaptic expression of long-term potentiation in the rat dentate gyrus demonstrated by variance-mean analysis. J Physiol (Lond) 518:121-130.

Represa A, Ben-Ari Y (2005) Trophic actions of GABA on neuronal development. Trends Neurosci 28:278-283.

Rosahl TW, Geppert M, Spillane D, Herz J, Hammer RE, Malenka RC, Sudhof
TC (1993) Short-term synaptic plasticity is altered in mice lacking synapsin I. Cell 75:661-670.

Rosahl TW, Spillane D, Missler M, Herz J, Selig DK, Wolff JR, Hammer RE, Malenka RC, Sudhof TC (1995) Essential functions of synapsins I and II in synaptic vesicle regulation. Nature 375:488-493.

Rosenmund C, Stevens CF (1996) Definition of the readily releasable pool of vesicles at hippocampal synapses. Neuron 16:1197-1207.

Ryan TA, Li L, Chin LS, Greengard P, Smith SJ (1996) Synaptic vesicle recycling in synapsin I knock-out mice. J Cell Biol 134:1219-1227.

Schneggenburger R, Meyer AC, Neher E (1999) Released fraction and total size of a pool of immediately available transmitter quanta at a calyx synapse. Neuron 23:399-409.

Schneggenburger R, Sakaba T, Neher E (2002) Vesicle pools and short-term synaptic depression: lessons from a large synapse. Trends Neurosci 25:206-212.

Silver RA, Momiyama A, Cull-Candy SG (1998) Locus of frequencydependent depression identified with multiple-probability fluctuation analysis at rat climbing fibre-Purkinje cell synapses. J Physiol (Lond) 510:881-902.

Siksou L, Rostaing P, Lechaire JP, Boudier T, Ohtsuka T, Fejtova A, Kao HT, Greengard P, Gundelfinger ED, Triller A, Marty S (2007) Threedimensional architecture of presynaptic terminal cytomatrix. J Neurosci 27:6868-6877.

Steinlein OK (2004) Genetic mechanisms that underlie epilepsy. Nat Rev Neurosci 5:400-408.

Sudhof TC, Czernik AJ, Kao HT, Takei K, Johnston PA, Horiuchi A, Kanazir SD, Wagner MA, Perin MS, De Camilli P, Greengard P (1989) Synapsins: mosaics of shared and individual domains in a family of synaptic vesicle phosphoproteins. Science 245:1474-1480.

Sun J, Bronk P, Liu X, Han W, Sudhof TC (2006) Synapsins regulate usedependent synaptic plasticity in the calyx of Held by a Ca ${ }^{2+} /$ calmodulindependent pathway. Proc Natl Acad Sci USA 103:2880-2885.

Takamori S, Holt M, Stenius K, Lemke EA, Gronborg M, Riedel D, Urlaub H, Schenck S, Brugger B, Ringler P, Muller SA, Rammner B, Grater F, Hub JS, De Groot BL, Mieskes G, Moriyama Y, Klingauf J, Grubmuller H, Heuser J, et al. (2006) Molecular anatomy of a trafficking organelle. Cell 127:831-846.

Takei Y, Harada A, Takeda S, Kobayashi K, Terada S, Noda T, Takahashi T, Hirokawa N (1995) Synapsin I deficiency results in the structural change in the presynaptic terminals in the murine nervous system. J Cell Biol 131:1789-1800.

Terada S, Tsujimoto T, Takei Y, Takahashi T, Hirokawa N (1999) Impairment of inhibitory synaptic transmission in mice lacking synapsin I. J Cell Biol 145:1039-1048.

Vaccaro P, Dente L, Onofri F, Zucconi A, Martinelli S, Valtorta F, Greengard P, Cesareni G, Benfenati F (1997) Anti-synapsin monoclonal antibodies: epitope mapping and inhibitory effects on phosphorylation and Grb2 binding. Brain Res Mol Brain Res 52:1-16.

Xu T, Bajjalieh SM (2001) SV2 modulates the size of the readily releasable pool of secretory vesicles. Nat Cell Biol 3:691-698.

Yamaguchi K, Tanaka M, Mizoguchi A, Hirata Y, Ishizaki H, Kaneko K, Miyoshi J, Takai Y (2002) A GDP/GTP exchange protein for the Rab3 small $\mathrm{G}$ protein family up-regulates a postdocking step of synaptic exocytosis in central synapses. Proc Natl Acad Sci USA 99:14536-14541.

Zucker RS, Regehr WG (2002) Short-term synaptic plasticity. Annu Rev Physiol 64:355-405. 